\title{
The Potential for use of Probiotics in Pediatric Irritable Bowel Syndrome and Inflammatory Bowel Disease
}

\author{
Stefano Guandalini
}

Published online: 15 June 2014

(C) Springer Science + Business Media New York 2014

\begin{abstract}
Controlled trials of probiotics in irritable bowel syndrome are promising, but most are limited by suboptimal design and small sample size. A recent report from the Rome foundation group included 32 randomized clinical trials of probiotics. Seventy-five percent of these studies (including the 4 pediatric ones) did show an improvement in symptoms, but the therapeutic gain over placebo was generally modest. The patients most benefitting from probiotics appear to be those with predominant diarrhea and those who have developed irritable bowel syndrome after an episode of gastroenteritis. A review focusing only on children with functional gastrointestinal disorders concluded that probiotics are more effective than placebo in the treatment of patients with abdominal pain-related functional disorders, but no effect on constipation was evident for any strain. In spite of a solid conceptual and experimental basis for successful use of probiotics in inflammatory bowel diseases (Crohn's disease and ulcerative colitis), research in humans has been overall quite limited and overall disappointing. To summarize current evidence, no probiotic has proven successful in Crohn's disease, while in ulcerative colitis data are more promising. In fact, a recent meta-analysis, that included 23 randomized controlled trials, concluded that there is evidence of efficacy for the probiotic mixture VSL\#3 in helping inducing and maintaining remission. In summary, for both irritable bowel syndrome and inflammatory bowel diseases, there is a definite need for well-designed, randomized clinical trials.
\end{abstract}

S. Guandalini $(\bowtie)$

Section of Gastroenterology, Hepatology and Nutrition

Department of Pediatrics, University of Chicago,

5841 S. Maryland Ave., MC 4065, Chicago, IL 60637, USA

e-mail: sguandalini@peds.bsd.uchicago.edu
Keywords Probiotics - Irritable bowel syndrome . Inflammatory bowel disease

\section{Introduction}

The interest in the intestinal microbiome during the past decade has exploded. With it, there has been a growing interest in the possible utilization of agents affecting it (prebiotics-intended to be a metabolic fuel for beneficial microorganisms - and probiotics—strains selected for their ability to survive the passage in the gastrointestinal tract and eventually provide health benefits to the host). Among the areas that appear more interesting is their potential application in 2 different but equally important conditions: irritable bowel syndrome (IBS) and inflammatory bowel disease (IBD), conditions that have a strong conceptual basis for a potentially useful implementation of their use. In spite of a large body of experimental evidence in various in vitro and animal models, randomized controlled clinical trials—especially in children — are not numerous. This mini review will examine the current status of the use of prebiotics and probiotics in children affected by either disorder.

\section{Probiotics in IBS}

Irritable bowel syndrome is a functional gastrointestinal disorder (FGID), and a very frequent one, as up to $25-50 \%$ of the children and teenagers that present to gastroenterology clinics are affected [1, 2]. Thus, IBS involves significant health care costs and has an important negative impact on quality of life and social functioning in many patients [3]. 
As well defined by the so-called Rome III criteria [4], IBS is characterized by the association of abdominal pain with a change in stool consistency or frequency in the absence of an organic cause.

The underlying pathophysiological mechanisms of this disorder are incompletely known, and it is quite likely that IBS is an umbrella term encompassing various etiological and pathogenetic factors eventually leading to the same syndromic phenotype [5]. Abnormal gastrointestinal motility, altered brain/gut function, low-grade inflammation, psychosocial disturbance, visceral hypersensitivity, and intestinal microbiota all appear to contribute substantially [6, 7] and probably in various degrees in different patients.

The potential role for probiotics in such syndrome is based on a number of observations. Alterations in the intestinal microbiota that have been described in patients with IBS versus non-IBS populations have been reported [8-10]; the development of IBS after a gastrointestinal infection is well documented ("post-infectious IBS") [11]; colonic fecal microbiota transplantation [12] has been utilized; and it is quite conceivable that microbiota may interfere with additional factors involved in the pathophysiology of IBS, such as visceral hypersensitivity and the brain-gut axis $[13,14,15 \bullet \cdot$.

In adults, several trials have been published, with sometimes conflicting results, although overall the outcomes have been quite promising $[6,16,17]$.

In children, fewer randomized clinical trials (RCTs) are available. Earlier investigations were not particularly encouraging: a Cochrane systematic review in 2009 [18] aiming at determining the efficacy of dietary interventions in recurrent abdominal pain and analyzing published RCT up to 2007, included two trials comparing supplementation with Lactobacillus GG against placebo [2, 19]. The authors concluded that there was lack of high-quality evidence that the probiotic supplementation could be considered effective in the management of children with recurrent abdominal pain.

Since then, new RCTs have been published, prompting interest in reevaluating the role of probiotics in the management of IBS in children.

In fact, a very recent systematic review and meta-analysis [20•] on the effect of different probiotics as a treatment for FGID in children and adolescents included 9 trials, 5 linked to the abdominal pain-related FGID [2, 19, 21-23], and 4 with defecation-related FGID [24-27].

The trials on abdominal pain used were Lactobacillus GG, Lactobacillus reuteri DSM 17 938, and the probiotic mixture VSL\#3. The meta-analysis concluded that the use of such probiotics significantly increased treatment success in children with abdominal pain-related FGID, especially children with IBS. In our double-blinded, placebo-controlled, crossover RCT [21], we found that the patients taking VSL\#3 compared with placebo experienced a significant $(P<0.05)$ improvement in the score for abdominal pain/discomfort from baseline to 6 week. More importantly, the global subjective relief of symptoms likewise improved more significantly for children on VSL\#3 than on placebo.

As for Lactobacillus reuteri DSM 17 938, it significantly decreased the intensity of abdominal pain [22, 28]; in Romano's study, its effect did persist after the removal of the probiotic, indicating a lasting effect of the supplementation [22].

Of interest, the two RCTs that assessed the effect of treatment with probiotics (Lactobacillus GG and VSL\#3) did not show any benefit of the intervention on the parameter of functional diarrhea $[2,21]$.

Four studies included in the meta-analysis assessed constipation-related FGID. The probiotics used were Lactobacillus GG associated with Lactulose, Lactobacillus casei rhamnosus Lcr35, Bifidobacterium longum, and Bifidobacterium lactis DN-173 010 [24-27]. The results were disappointing, with no evidence that probiotics are more effective than placebo in overall outcome of treatment or in increasing defecation frequency in constipated children and with inconsistent data on the improvement in stool consistency [25, 26].

Thus, in summary, it appears that at present, some strains of probiotics offer a modest but significant benefit in the treatment of FGID in children. Considering their general safety and the lack of viable and safe treatment options for these frequent and annoying conditions, they may have a potentially interesting role. Clearly, a long way is in front of us as we need to define strain selections, the choice of single organisms versus mixtures, dosage, safety and especially long-term efficacy, and tolerability.

\section{Probiotics in IBD}

The introduction of biological agents has markedly improved our ability to treat patients with the chronic, incurable inflammatory disorders grouped under the name of IBD, namely Crohn's disease, ulcerative colitis, and indeterminate colitis. However, improved clinical efficacy has come at the cost of increased risks, including malignancies $[29,30]$. Thus, it is not surprising that the use of "alternative" remedies is very frequent in patients with IBD, and especially in children [31]. In this regard, the possible use of probiotics appears as a natural consideration, especially at the light of the fact that the intestinal microbiota does play an important role in IBD [32•].

In fact, on one side probiotics display features, such as anti-inflammatory action and enhancement of the gut barrier, that seem logically suited to be helpful in IBD; and on the other, there is convincing evidence that the inflammation in IBD results from an altered mucosal immune response to luminal bacterial antigens, thus suggesting that 
the use of live microorganisms may have a positive impact on shifting the microbial balance of the microbiota. Support for this hypothesis comes also from a study showing that children with Crohn's disease treated with exclusive and partial polymeric enteral nutrition and experiencing remission with this regimen also showed profound changes in their microbiota, as measured by temperature gradient gel electrophoresis [33].

In the last few years, the IBD microbiota-both in CD and in $\mathrm{UC}$ - has been found to be less diverse and to have a different composition compared with healthy controls, and differences have also been found between the microbiota composition in UC versus CD patients [34-36] ), to the point that such "dysbiosis" has been called one of the major factors involved in the course of inflammation in IBD [37•].

A large body of data shows efficacy of several probiotic strains in ameliorating the inflammation induced in various animal models of colitis, considered to some extent as models of IBD, and especially ulcerative colitis. Some examples include:

In mice models of colitis: Enterococcus faecalis, $L$. acidophilus, C. butyricum and B. adolescentis [38], VSL\#3 [39, 40], E. coli M-17 [41], Lactobacillus salivarius [42]; a mixture of Lactobacillus strains-Lactobacillus GG, L. plantarum, L. casei and L. lactis, Bifidobacterium bifidum, B. infantis, B. lactis and B. Adolescentis [43]; Lactobacillus fermentum [44], Saccharomyces boulardii [45], Lactobacillus casei [46, 47] Bifidobacterium lactis and Lactobacillus acidophilus [47].

In rat model of colitis, other strains have been found effective: Lactobacillus GG and a mixture of Streptococcus thermophilus, Lactobacillus acidophilus, and Bifidobacterium longum [48], VSL\#3 [49]; Lactobacillus plantarum HY115 and L. brevis [50] ); Lactobacillus reuteri and Lactobacillus fermentum [51]. VSL\#3 results in a decrease of tumor necrosis factor-a, IL-6 and an increase of IL-10, which may due to exert the anti-inflammatory activity by inhibiting PI3K/Akt and NF-kB pathway [52]

In spite of the strength of the conceptual basis for the beneficial use of probiotics, and of the extensive experimental data in animal models, the existing literature-and in particularly concerning papers reporting $\mathrm{RCT}-\mathrm{on}$ the use of probiotics in IBD in adults and even more so in children is indeed extremely limited. The analysis will be limited to papers reporting RCT with a comparator, either a placebo or an accepted standard therapy.

\section{Crohn's Disease}

A 2012 meta-analysis [36] detected only 3 small uncontrolled studies and one placebo-controlled trial in adults patients with active $\mathrm{CD}$. The trial reported a lower relapse rate after 12 months receiving E. coli Nissle 1917 added to prednisolone therapy [53].

The same review included 4 studies in patients with inactive CD: two using Lactobacillus GG [54, 55] and two using Lactobacillus johnsonii [56, 57]. None of them could demonstrate that probiotics were more effective than placebo in preventing relapses.

A small study published in 2000 found a significantly lower relapse rate in CD when Saccharomyces boulardii was combined with mesalazine [58]; however, a subsequent larger randomized, double-blind placebo-controlled trial utilizing the same probiotic yeast showed no significant difference in the frequency of relapses in the Saccharomyces boulardii group (47.5\%) compared to the placebo one $(53.2 \%)$; the time to relapse was also not statistically different [59••].

A 2014 meta-analysis [60] included 23 randomized controlled trials (only 3, however, conducted in children) published up to 2011, with a total of 1763 participants: 12 in UC, 4 in "pouchitis," and 7 in CD.

None of the trials $[53,55,61]$ that were the remission/ response rates in acute $\mathrm{CD}$ were reported, showed any significant benefit in favor of probiotics supplementation $(P=0.35, R R=0.89)$.

As for efficacy of probiotics in maintaining remission, an obvious major goal in the potential use of probiotics, 7 RCTs (including only one in children, reported in more detail below) examined the frequency of and timing to clinical relapses, finding that there was actually again no difference between the probiotics-supplemented patients and the control ones $(P=0.71, R R=1.09)$.

In children, a randomized, placebo-controlled trial of the probiotic Lactobacillus GG [62] with the aim of comparing the remission in patients that receive Lactobacillus GG added to standard therapy was unable to demonstrate that this probiotic would prolong remission time in patients with $\mathrm{CD}$ already in remission on standard therapy. In fact, the time to relapse and proportion of patients relapsing was essentially identical in both Lactobacillus GG and placebo groups.

\section{Ulcerative Colitis}

A more favorable picture is obtained when looking at the use of probiotics in UC. As mentioned, of the 23 RCTs included in the 2014 meta-analysis [60], twelve were realized in UC patients.

Nine studies analyzed the remission/response in acute UC, utilizing Bifidobacteria, E. Coli, and VSL\#3: while the overall analysis of these RCTs showed significant benefit from the use of probiotics, the sub-analysis based on probiotic utilized documented such significant effect only for VSL\#3 [63••, 64-67]. 
As for their efficacy in maintaining remission once pharmacologically obtained, the analysis included 5 trials in UC and there was no advantage of probiotics compared with placebo (RR 0.89, $95 \%$ CI 0.66-1.21). However, the only pediatric trial, conducted with VSL\#3 [63••], showed efficacy of the probiotic ( $R R \quad 0.29$, $95 \%$ CI $0.10-0.83$ ). The trial included 29 children, with a recent diagnostic of UC who received initially prednisone and mesalamine plus either placebo or VSL\#3 for induction; once remission had been obtained, they were continued on mesalamine plus either placebo or VSL\#3 for maintenance. The patients were evaluated at 1, 2, 6 months, and 1 year after diagnosis or at the time of relapse. An endoscopy was additionally performed at the time of diagnosis and then repeated at 6 , 12 months or at the time of relapse. As mentioned, not only the remission rate, but also the relapse rates were better in the patients treated with VSL\#3 vs placebo; furthermore, endoscopic and histological scores were significantly better in the VSL\#3 group than in the placebo group $(P<0.05)$.

A recent pediatric trial included 31 children with mild to moderate ulcerative proctitis/proctosigmoiditis with mild to moderate disease activity [68•]; they received enema solution with $L$. reuteri ATCC 55730 or placebo during 8 weeks added to the oral treatment with mesalazine. A clinical, endoscopic, histological, and immune evaluation (IL-10, IL-1b, TNFa, IL-8) was performed. Clinical and endoscopic improvements were shown to be better in the probiotic group, and the histological score was significantly decreased in the $L$. reuteri group $(P<0.01)$. As for the cytokines evaluation, a significant increase in the mucosal expression levels of IL-10 and a significant decrease in the levels of IL-1b, TNFa, and IL-8 mucosal expression levels $(P<0.01)$ were documented only in the $L$. reuteri group.

\section{Conclusion}

Both IBS and IBD, as conditions originating at least in part by an abnormal interaction between microbiota and the host immune system, are potential candidate to be addressed by interventions aiming at normalizing such imbalance. In this regard, probiotics have been studied extensively in various animal models of IBS as well as of IBD, with encouraging results. Their clinical applications in children with these conditions, however, are still limited, and in many cases hampered by limited size or short duration studies. At the present time, the following conclusions appear valid:

1. IBS. Some probiotic strains, namely the probiotic mixture VSL\#3, Lactobacillus GG, and possibly Lactobacillus reuteri DSM 17 938, have shown some efficacy in improving overall symptoms and especially abdominal pain in children with IBS. Considering the limited role for safe and effective pharmacological agents, they may represent useful options.

2. IBD. To date, there is no evidence of efficacy for any strain in pediatric (or adult) Crohn's disease. It is, however, quite possible that, given the array of genotypes and phenotypes of Crohn's disease, the investigators have yet to identify the specific probiotics that may be beneficial in specific forms of this chronic, multiform inflammatory process. As for ulcerative colitis, there is promising evidence of efficacy for the probiotic mixture VSL\#3.

Clearly, progress in the field will require on one side the better identification of probiotic strains, and their testing in adequately powered, long-term pluricentric RCT.

Disclosure Stefano Guandalini declares that he has no conflict of interest.

Human and Animal Rights and Informed Consent This article does not contain any studies with human or animal subjects performed by any of the authors.

\section{References}

Papers of particular interest, published recently, have been highlighted as:

- Of importance

- Of major importance

1. Everhart JE, Renault PF. Irritable bowel syndrome in office-based practice in the United States. Gastroenterology. 1991;100(4): 998-1005.

2. Bausserman M, Michail S. The use of Lactobacillus GG in irritable bowel syndrome in children: a double-blind randomized control trial. J Pediatr. 2005;147(2):197-201.

3. Spiller R, et al. Guidelines on the irritable bowel syndrome: mechanisms and practical management. Gut. 2007;56(12):1770-98.

4. Rasquin A, et al. Childhood functional gastrointestinal disorders: child/adolescent. Gastroenterology. 2006;130(5):1527-37.

5. Longstreth GF, et al. Functional bowel disorders. Gastroenterology. 2006;130(5):1480-91.

6. Kruis W, et al. A double-blind placebo-controlled trial to study therapeutic effects of probiotic Escherichia coli Nissle 1917 in subgroups of patients with irritable bowel syndrome. Int $\mathrm{J}$ Colorectal Dis. 2012;27(4):467-74.

7. Quigley EM, Flourie B. Probiotics and irritable bowel syndrome: a rationale for their use and an assessment of the evidence to date. Neurogastroenterol Motil. 2007;19(3):166-72.

8. Kassinen A, et al. The fecal microbiota of irritable bowel syndrome patients differs significantly from that of healthy subjects. Gastroenterology. 2007;133(1):24-33.

9. Carroll IM, et al. Alterations in composition and diversity of the intestinal microbiota in patients with diarrhea-predominant irritable bowel syndrome. Neurogastroenterol Motil. 2012;24(6): 521-30 e248. 
10. Saulnier DM, et al. Gastrointestinal microbiome signatures of pediatric patients with irritable bowel syndrome. Gastroenterology. 2011;141(5):1782-91.

11. Saps M, et al. Post-infectious functional gastrointestinal disorders in children. J Pediatr. 2008;152(6):812-6 816 e1.

12. DuPont HL. Review article: evidence for the role of gut microbiota in irritable bowel syndrome and its potential influence on therapeutic targets. Aliment Pharmacol \& Ther. 2014;39(10):1033-42.

13. Johnson AC, Greenwood-Van Meerveld B, McRorie J. Effects of Bifidobacterium infantis 35624 on post-inflammatory visceral hypersensitivity in the rat. Dig Dis Sci. 2011;56(11):3179-86.

14. Collins SM, Surette M, Bercik P. The interplay between the intestinal microbiota and the brain. Nat Rev Microbiol. 2012; 10(11):735-42.

15. • De Palma G., et al. The Microbiota-Gut-Brain axis in gastrointestinal disorders: stressed bugs, stressed brain or both? J Physiol. 2014. Updated review on the literature on the role of gut microbiota in gut homeostasis and its link with mechanisms that influence behavior.

16. Camilleri M. Management of the irritable bowel syndrome. Gastroenterology. 2001;120(3):652-68.

17. McFarland LV, Dublin S. Meta-analysis of probiotics for the treatment of irritable bowel syndrome. World J Gastroenterol. 2008;14(17):2650-61.

18. Huertas-Ceballos AA, et al. Dietary interventions for recurrent abdominal pain (RAP) and irritable bowel syndrome (IBS) in childhood. Cochrane Database Syst Rev. 2009;21(1):Cd003019.

19. Gawronska A, et al. A randomized double-blind placebo-controlled trial of Lactobacillus $\mathrm{GG}$ for abdominal pain disorders in children. Aliment Pharmacol Ther. 2007;25(2):177-84.

20. - Korterink JJ, et al. Probiotics for childhood functional gastrointestinal disorders: a systematic review and meta-analysis. Acta Paediatr. 2014;103(4):365-72. The most recent systematic review and meta-analysis on probiotics in childhood FGID.

21. Guandalini S, et al. VSL\#3 improves symptoms in children with irritable bowel syndrome: a multicenter, randomized, placebocontrolled, double-blind, crossover study. J Pediatr Gastroenterol Nutr. 2010;51(1):24-30.

22. Romano $\mathrm{C}$, et al. Lactobacillus reuteri in children with functional abdominal pain (FAP). J Paediatr Child Health. 2010. doi:10. 1111/j.1440-1754.2010.01797.x.

23. Francavilla $\mathrm{R}$, et al. Randomised clinical trial: Lactobacillus reuteri DSM 17938 vs. placebo in children with acute diarrhoea-a double-blind study. Aliment Pharmacol Ther. 2012;36(4):363-9.

24. Banaszkiewicz A, Szajewska H. Ineffectiveness of Lactobacillus GG as an adjunct to lactulose for the treatment of constipation in children: a double-blind, placebo-controlled randomized trial. J Pediatr. 2005;146(3):364-9.

25. Bu LN, et al. Lactobacillus casei rhamnosus Lcr35 in children with chronic constipation. Pediatr Int. 2007;49(4):485-90.

26. Guerra PV, et al. Pediatric functional constipation treatment with Bifidobacterium-containing yogurt: a crossover, double-blind, controlled trial. World J Gastroenterol. 2011;17(34):3916-21.

27. Tabbers MM, et al. Effect of the consumption of a fermented dairy product containing Bifidobacterium lactis DN-173 010 on constipation in childhood: a multicentre randomised controlled trial (NTRTC: 1571). BMC Pediatr. 2009;9:22.

28. Francavilla R, et al. A randomized controlled trial of Lactobacillus GG in children with functional abdominal pain. Pediatrics. 2010;126(6): e1445-52.

29. Biancone L, et al. Treatment with biologic therapies and the risk of cancer in patients with IBD. Nat Clin Pract Gastroenterol Hepatol. 2007;4(2):78-91.

30. de Vries HS, van Oijen MG, de Jong DJ. Serious events with infliximab in patients with inflammatory bowel disease: a 9-year cohort study in the Netherlands. Drug Saf. 2008;31(12):1135-44.
31. Wong AP, et al. Use of complementary medicine in pediatric patients with inflammatory bowel disease: results from a multicenter survey. J Pediatr Gastroenterol Nutr. 2009;48(1):55-60.

32. - Scharl M, Rogler G. Inflammatory bowel disease pathogenesis: what is new?. Curr Opin Gastroenterol. 2012; 28(4):p. 301-9. Review on new pathogenetic mechanisms involved in inflammatory bowel disease, showing how epigenetic changes triggered by environmental factors probably contribute to heritability and also affect the composition of the gut microbiome and its interaction with the mucosal immune system.

33. Lionetti $\mathrm{P}$, et al. Enteral nutrition and microflora in pediatric Crohn's disease. JPEN J Parenter Enter Nutr. 2005;29(4):S173-5 discussion S175-8, S184-8.

34. Dicksved J, et al. Molecular analysis of the gut microbiota of identical twins with Crohn's disease. ISME J. 2008;2(7):716-27.

35. Martinez C, et al. Unstable composition of the fecal microbiota in ulcerative colitis during clinical remission. Am J Gastroenterol. 2008;103(3):643-8.

36. Jonkers D, et al. Probiotics in the management of inflammatory bowel disease: a systematic review of intervention studies in adult patients. Drugs. 2012;72(6):803-23.

37. - Hold GL, et al. Role of the gut microbiota in inflammatory bowel disease pathogenesis: what have we learnt in the past 10 years?. World J Gastroenterol. 2014; 20(5):p. 1192-210. Updated review on advances in our understanding of microbial involvement in IBD pathogenesis over the past 10 years.

38. Chen LL, et al. Therapeutic effects of four strains of probiotics on experimental colitis in mice. World J Gastroenterol. 2009;15(3): 321-7.

39. Soo I, et al. VSL\#3 probiotic upregulates intestinal mucosal alkaline sphingomyelinase and reduces inflammation. Can $\mathrm{J}$ Gastroenterol. 2008;22(3):237-42.

40. Mennigen R, et al. Probiotic mixture VSL\#3 protects the epithelial barrier by maintaining tight junction protein expression and preventing apoptosis in a murine model of colitis. Am J Physiol Gastrointest Liver Physiol. 2009;296(5):G1140-9.

41. Fitzpatrick LR, et al. In vitro and in vivo effects of the probiotic Escherichia coli strain M-17: immunomodulation and attenuation of murine colitis. Br J Nutr. 2008;100(3):530-41.

42. Feighery LM, et al. Effects of Lactobacillus salivarius 433118 on intestinal inflammation, immunity status and in vitro colon function in two mouse models of inflammatory bowel disease. Dig Dis Sci. 2008;53(9):2495-506.

43. Nanda Kumar NS, et al. Probiotic administration alters the gut flora and attenuates colitis in mice administered dextran sodium sulfate. J Gastroenterol Hepatol. 2008;23(12):1834-9.

44. Zoumpopoulou G, et al. Lactobacillus fermentum ACA-DC 179 displays probiotic potential in vitro and protects against trinitrobenzene sulfonic acid (TNBS)-induced colitis and Salmonella infection in murine models. Int J Food Microbiol. 2008;121(1): $18-26$.

45. Wu X, et al. Saccharomyces boulardii ameliorates Citrobacter rodentium-induced colitis through actions on bacterial virulence factors. Am J Physiol Gastrointest Liver Physiol. 2008;294(1): G295-306.

46. Chung YW, et al. Lactobacillus casei prevents the development of dextran sulphate sodium-induced colitis in Toll-like receptor 4 mutant mice. Clin Exp Immunol. 2008;151(1):182-9.

47. Peran L, et al. A comparative study of the preventative effects exerted by three probiotics, Bifidobacterium lactis, Lactobacillus casei and Lactobacillus acidophilus, in the TNBS model of rat colitis. J Appl Microbiol. 2007;103(4):836-44.

48. Amit-Romach E, Uni Z, Reifen R. Therapeutic potential of two probiotics in inflammatory bowel disease as observed in the trinitrobenzene sulfonic acid model of colitis. Dis Colon Rectum. 2008;51(12):1828-36. 
49. Fitzpatrick LR, et al. Effects of the probiotic formulation VSL\#3 on colitis in weanling rats. J Pediatr Gastroenterol Nutr. 2007; 44(5):561-70.

50. Lee HS, et al. Lactic acid bacteria inhibit proinflammatory cytokine expression and bacterial glycosaminoglycan degradation activity in dextran sulfate sodium-induced colitic mice. Int Immunopharmacol. 2008;8(4):574-80.

51. Peran L, et al. A comparative study of the preventative effects exerted by two probiotics, Lactobacillus reuteri and Lactobacillus fermentum, in the trinitrobenzenesulfonic acid model of rat colitis. Br J Nutr. 2007;97(1):96-103.

52. Dai $\mathrm{C}$, et al. VSL\#3 probiotics exerts the anti-inflammatory activity via PI3k/Akt and NF-kappaB pathway in rat model of DSS-induced colitis. Mol Cell Biochem. 2013;374(1-2):1-11.

53. Malchow HA. Crohn's disease and Escherichia coli. A new approach in therapy to maintain remission of colonic Crohn's disease? J Clin Gastroenterol. 1997;25(4):653-8.

54. Prantera $\mathrm{C}$, et al. Ineffectiveness of probiotics in preventing recurrence after curative resection for Crohn's disease: a randomised controlled trial with Lactobacillus GG. Gut. 2002;51(3):405-9.

55. Schultz M, et al. Lactobacillus GG in inducing and maintaining remission of Crohn's disease. BMC Gastroenterol. 2004;4:5.

56. Marteau P, et al. Ineffectiveness of Lactobacillus johnsonii LA1 for prophylaxis of postoperative recurrence in Crohn's disease: a randomised, double blind, placebo controlled GETAID trial. Gut. 2006;55(6):842-7.

57. Van Gossum A, et al. Multicenter randomized-controlled clinical trial of probiotics (Lactobacillus johnsonii, LA1) on early endoscopic recurrence of Crohn's disease after lleo-caecal resection. Inflamm Bowel Dis. 2007;13(2):135-42.

58. Guslandi M, et al. Saccharomyces boulardii in maintenance treatment of Crohn's disease. Dig Dis Sci. 2000;45(7):1462-4.

59. $\bullet$ Bourreille A, et al. Saccharomyces boulardii does not prevent relapse of Crohn's disease. Clin Gastroenterol Hepatol. 2013; 11(8):p. 982-7. The most recent negative study on a probiotic in Crohn's disease. A well-designed RCT on 165 adult patients followed for up to 1 year. A good example of why even negative studies need to be published.
60. Shen J, Zuo ZX, Mao AP. Effect of probiotics on inducing remission and maintaining therapy in ulcerative colitis, Crohn's disease, and pouchitis: meta-analysis of randomized controlled trials. Inflamm Bowel Dis. 2014;20(1):21-35.

61. Steed H, et al. Clinical trial: the microbiological and immunological effects of synbiotic consumption-a randomized doubleblind placebo-controlled study in active Crohn's disease. Aliment Pharmacol Ther. 2010;32(7):872-83.

62. Bousvaros A, et al. A randomized, double-blind trial of Lactobacillus GG versus placebo in addition to standard maintenance therapy for children with Crohn's disease. Inflamm Bowel Dis. 2005;11(9):833-9.

63. $\bullet$ Miele E, et al. Effect of a probiotic preparation (VSL\#3) on induction and maintenance of remission in children with ulcerative colitis. Am J Gastroenterol. 2009; 104(2):p. 437-43. The best RCT so far showing evidence of efficacy of a probiotic in ulcerative colitis in children.

64. $\mathrm{Ng} \mathrm{SC}$, et al. Immunosuppressive effects via human intestinal dendritic cells of probiotic bacteria and steroids in the treatment of acute ulcerative colitis. Inflamm Bowel Dis. 2010;16(8): 1286-98.

65. Sood A, et al. The probiotic preparation, VSL\#3 induces remission in patients with mild-to-moderately active ulcerative colitis. Clin Gastroenterol Hepatol. 2009;7(11):1202-9 1209 e1.

66. Tursi A, et al. Low-dose balsalazide plus a high-potency probiotic preparation is more effective than balsalazide alone or mesalazine in the treatment of acute mild-to-moderate ulcerative colitis. Med Sci Monit. 2004;10(11):Pi126-31.

67. Tursi A, et al. Treatment of relapsing mild-to-moderate ulcerative colitis with the probiotic VSL\#3 as adjunctive to a standard pharmaceutical treatment: a double-blind, randomized, placebocontrolled study. Am J Gastroenterol. 2010;105(10):2218-27.

68. - Oliva S. et al. Randomised clinical trial: the effectiveness of Lactobacillus reuteri ATCC 55730 rectal enema in children with active distal ulcerative colitis. Aliment Pharmacol Ther. 2012; 35(3):p. 327-34. An interesting therapeutic option for children with ulcerative colitis limited to the recto-sigmoid area. 